\title{
MEASURING THE DIELECTRIC PROPERTIES OF TUMOR AND BREAST PHANTOMS USED IN THE MICROWAVE FREQUENCY RANGE
}

\author{
Eva Maniakova1, Dagmar Faktorova²
}

\begin{abstract}
INTRODUCTION: This article deals with measurement of dielectric properties (relative permittivity and conductivity) of phantoms, specifically a tumor phantom and a breast phantom. We focused on the waveguide and resonance methods for the measurement of dielectric properties. The article describes the principle of these methods, and also the production process of a breast phantom and a tumor phantom. These phantoms can be used for measurements in the microwave frequency range, $8-12 \mathrm{GHz}$.
\end{abstract}

OBJECTIVE: The study's objective was to design a tumor phantom and a breast phantom, and to measure their dielectric properties. These properties must simulate human tissue.

METHODS: To measure dielectric properties of human tissue, phantoms were designed using the waveguide Hippel's method and the resonance method with a cavity resonator.

RESULTS: The aim of this work was to create the phantoms that would have properties comparable to those of real tissues. Results of measurement are shown as frequency dependence of relative permittivity and conductivity for breast, breast phantom, tumor, and tumor phantom.

UDC Classification: 601 DOI: http://dx.doi.org/10.12955/cbup.v4.826

Keywords: dielectric properties, breast phantom, tumor phantom, permittivity, conductivity.

\section{Introduction}

Biological tissues are considered to have a dielectric loss factor. The relationships that describe the response of particles with an electrical charge in an external magnetic field are used to describe the interaction of electromagnetic waves with biological material. Dielectric properties of tissues are influenced by parameters such as frequency, temperature, water content in tissue, and others. The basic dielectric properties, which will be the subject of our interest, are relative permittivity and conductivity (Kumar \& Raju, 2014).

Permittivity $(\varepsilon)$ is a physical quantity that describes electrical (e.g, the ability of polarization) and insulating properties of material, especially the relationship between vectors of an electrical field. The permittivity, $\varepsilon$, in a relationship, is defined as the product of the vacuum permittivity $\left(\varepsilon_{0}=8.85 \times 10^{-12}\right.$ $\mathrm{F} / \mathrm{m})$ and as relative permittivity $\left(\varepsilon_{\mathrm{r}}\right)$ of a given environment, which is generally tensor quantity. The permittivity of dielectrics is frequency and temperature dependent (Chaudhary, Mishra, Swarup, \& Thomas, 1984).

The microwave methods used to measure dielectric properties of material include methods of measurement in free space, resonance methods using cavity resonators, measurements in waveguides of various shapes, and measurements using micro strip lines. In our work we use two methods: a resonant and a waveguide method (Faktorova, 2010; Faktorova, 2014).

\section{The Tissue Phantom Production}

In this article, we focused on the design and production of phantoms with a simple procedure for preparation of available material. These phantoms will be used for measurement in high-frequency electromagnetic field in the area of microwaves $(8-12 \mathrm{GHz})$. We focused on the production of breast tissue and tumor tissue phantoms. To produce the breast tissue phantom, we used demineralized water, agarose, corn oil, and neutral detergent and for the tumor tissue phantom, we used demineralized water, agarose, ethanol, and sodium chloride $(\mathrm{NaCl})$. We used the mixing procedure described in (Ortega-Palacios, Leija, Vera and Cepeda, 2010). The final phantoms, after solidification, are shown in Figure 1.

\footnotetext{
${ }^{1}$ Eva Maniakova, Department of Measurement and Applied Electrical Engineering, Faculty of Electrical Engineering, University of Zilina, eva.maniakova@ fel.uniza.sk

${ }^{2}$ Dagmar Faktorova, Department of Measurement and Applied Electrical Engineering, Faculty of Electrical Engineering, University of Zilina, dagmar.faktorova@fel.uniza.sk
} 
Figure 1: Tumor phantom and breast phantom

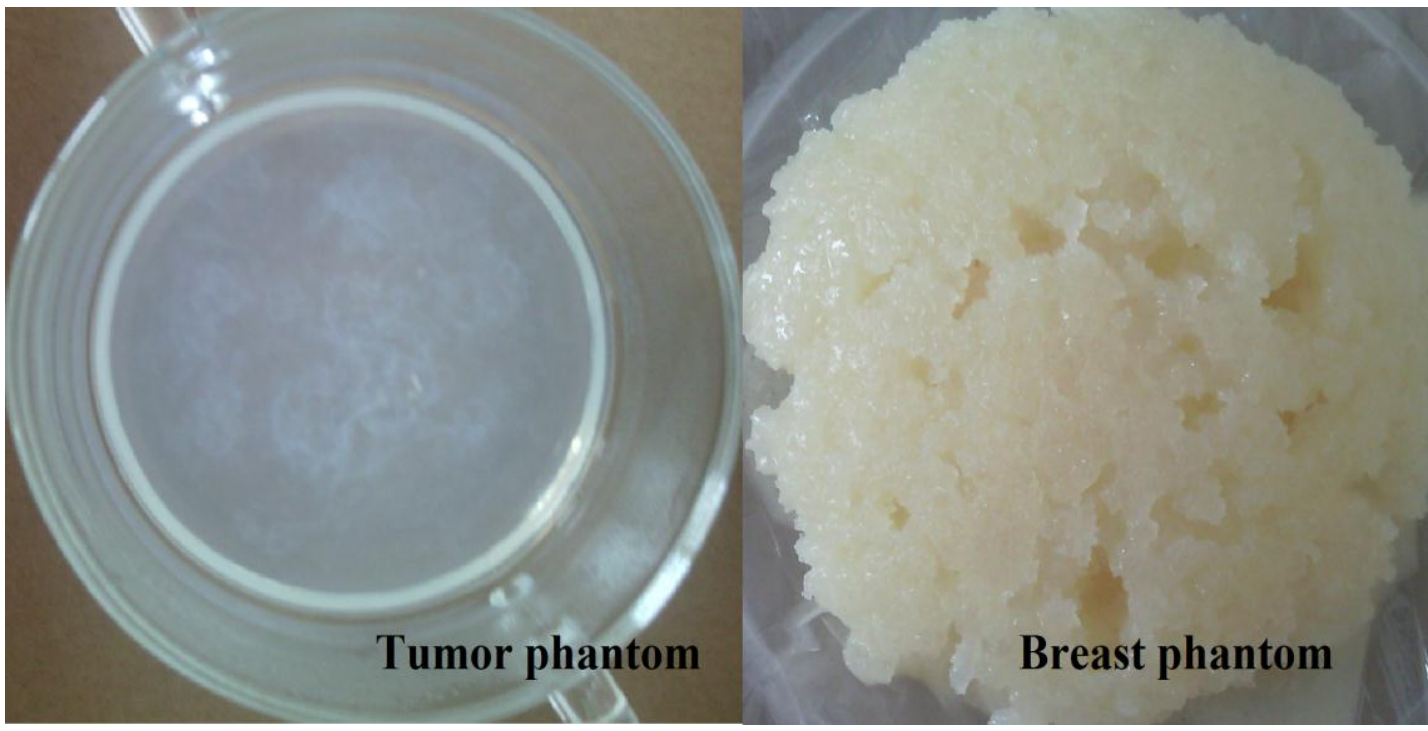

Source: Authors

\section{Measurement of Dielectric Properties}

The next step determined whether the dielectric properties of the phantoms were identical or similar to those of the chosen human tissue, particularly breast and tumor tissue. The "real" values were considered to be those listed in (Lazebnik, Popovic, McCartney, Watkins and Lindstrom, 2007).

To measure dielectric properties, we used two methods. The waveguide Hippel`s method, which is generally applicable, sufficiently accurate, and measures permittivity of medium loss materials, is suitable for measuring dielectric properties of homogenous structures. We used this approach to measure the dielectric properties of the tumor tissue phantom. However, the breast tissue phantom is heterogeneous, and thus, for the breast tissue phantom, we chose the resonance method, as it is the more accurate method in this case, and involved a cavity resonator to measure dielectric properties. This method requires microscopic samples to determine such properties (Faktorova, 2014; Tofighi \& Daryoush, 2006; Venkatesh \& Raghavan, 2005).

\section{Waveguide Hippel`s Method}

The input complex impedance, $\dot{Z}_{S}$ of dielectric waveguide:

$$
\dot{Z}_{S}=\dot{Z}_{d} \operatorname{tgh} \dot{\gamma} d
$$

where $\dot{Z}_{\mathrm{d}}$ is the complex impedance of dielectric waveguide (Equation 2); $\dot{\gamma}$ the propagation constant derived from $\alpha+\mathrm{j} \beta$, where $\alpha$ is the loss factor and $\beta$ is the phase constant; and $d$ is the length of the sample places in the waveguide, tgh is hyperbolic tangent (Faktorova, 2014; Tofighi \& Daryoush, 2006; Venkatesh \& Raghavan, 2005).

The complex impedance of waveguide:

$$
\dot{Z}_{d}=\frac{377}{\sqrt{\varepsilon_{r}-\left(\frac{\lambda}{\lambda_{c}}\right)^{2}}}
$$

where $\lambda$ is the wavelength in free space and $\lambda_{c}$ is critical length for a given waveguide.

The relative permittivity, $\varepsilon_{\mathrm{r}}$, of the sample can be calculated using the formula: 


$$
\varepsilon_{r}=\frac{\left(\frac{2 \pi}{\lambda_{c}}\right)^{2}+\beta^{2}-\alpha^{2}}{\left(\frac{2 \pi}{\lambda}\right)^{2}}
$$

\section{Resonance Methods Using Cavity Resonator}

Resonance methods are based on placing dielectric samples of small dimensions into the resonance cavity (filled partially or completely with the sample), where the sample causes the shift of resonance frequency, $f_{\mathrm{r}}$ (bound to $\varepsilon^{\prime}$ ), and the change in the quality factor, Q (bond to $\varepsilon^{\prime \prime}$ ).

Based on the measurement of the resonance frequency and quality factor, with empty resonance cavity $\left(f_{\mathrm{r} 1}, Q_{1}\right)$ and cavity filled with dielectric sample $\left(f_{\mathrm{r} 2}, Q_{2}\right)$, the permittivity of samples in a case of the resonator being completely filled with sample, can be expressed using the formula:

$$
\varepsilon^{\prime}=\left(\frac{f_{r 1}}{f_{r 2}}\right)^{2},
$$

and in case of the resonator only partially filled with the sample using formula:

$$
\varepsilon^{\prime \prime}=\frac{1}{2}\left(\frac{f_{r 1}}{f_{r 2}}-1\right) \frac{V_{c}}{V_{S}}-1
$$

where $V_{\mathrm{C}}$ describes the volume of empty resonance cavity and $V_{\mathrm{S}}$ the volume of sample. The size of the cavity and the dimensions of the sample are limited by the resonance frequency. Thus, these two methods were suitable for measuring dielectric parameters of small samples in comparison to cavity dimensions (Faktorova, 2014; Tofighi \& Daryoush, 2006; Venkatesh \& Raghavan, 2005).

\section{Results}

The aim of our work was to create tissue phantoms with properties comparable to those of real tissues. The frequency dependence of relative permittivity for both a tumor and a tumor phantom is shown in Figure 2 and that of conductivity for both the tumor and the tumor phantom in Figure 3. The frequency dependence of relative permittivity for both the breast and the breast phantom is shown in Figure 4 and that of conductivity for a breast and a breast phantom in Figure 5.

Figure 2: Frequency dependence of relative permittivity of the tumor and the tumor phantom

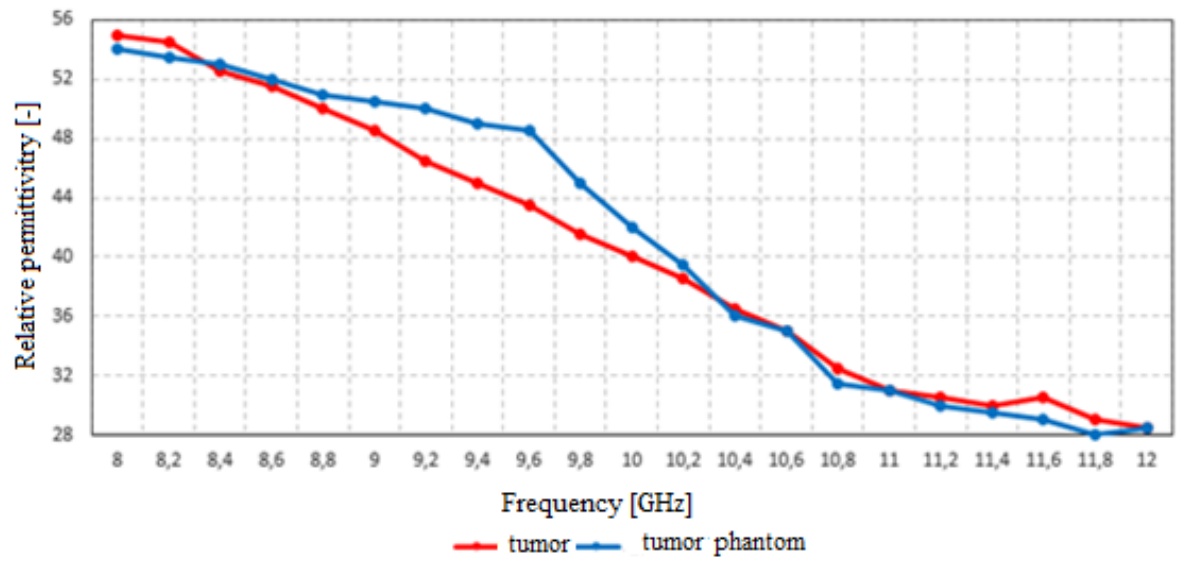

Source: Authors 
Figure 3: Frequency dependence of conductivity of the tumor and the tumor phantom

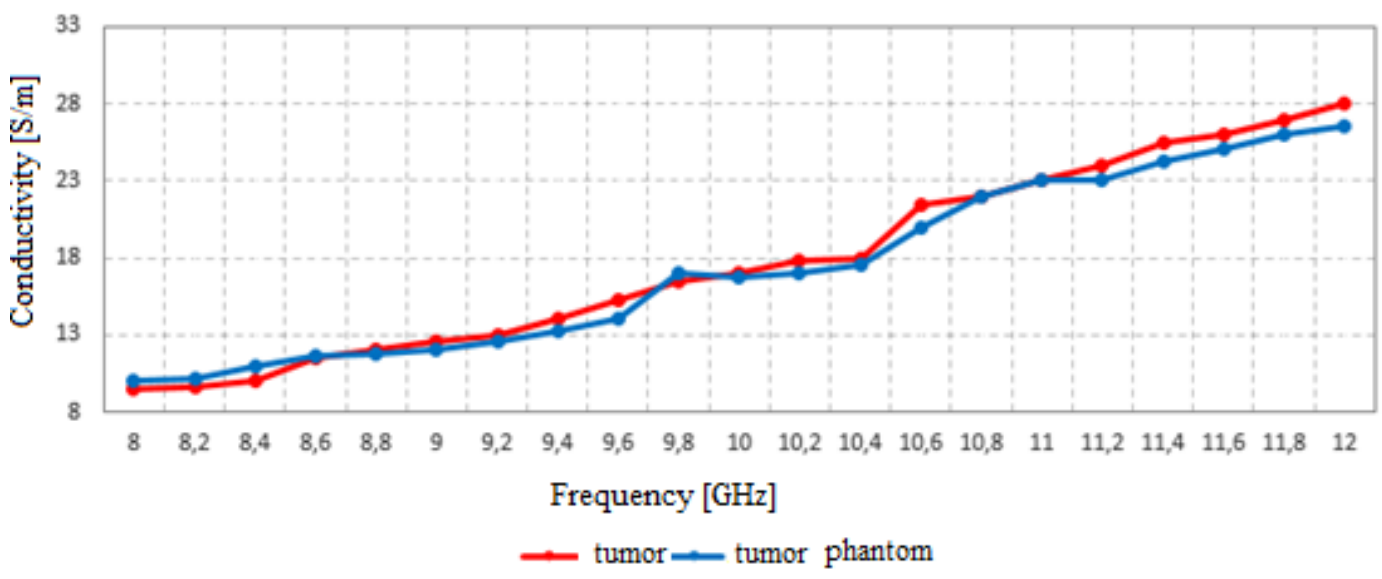

Source: Authors

Figure 4: Frequency dependence of relative permittivity of the breast and the breast phantom



$\longrightarrow$ breast $\longrightarrow$ breast phantom

Source: Authors

Figure 5: Frequency dependence of conductivity of the breast and the breast phantom

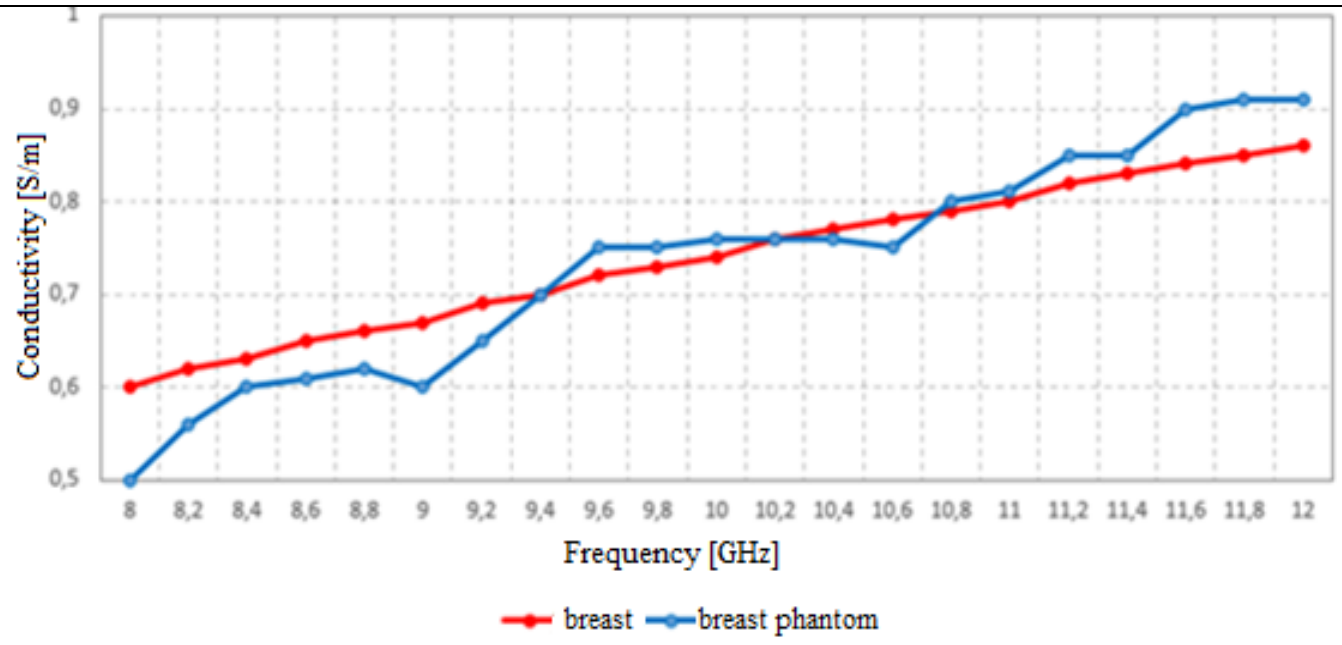

Source: Authors 


\section{Conclusion}

The resulting graphs show that we were able to create tissue phantoms with basic dielectric properties (relative permittivity and conductivity) similar to those of tissue. Hence, it is possible to further the use of such phantoms for measurements using the microwave frequency range.

\section{References}

Faktorova, D. (2010, November). Malignant tissue phantom dielectric properties investigation at microwave frequencies. In Applied Sciences in Biomedical and Communication Technologies (ISABEL), 2010 3rd International Symposium on (pp. 1-5). IEEE.

Faktorova, D. (2014) Basics of microwave measurements. Zilina: EDIS - Publishers ZU. ISBN 978-80-554-0870-5.

Chaudhary, S. S., Mishra, R. K., Swarup, A., \& Thomas, J. M. (1984). Dielectric properties of normal \& malignant human breast tissues at radiowave \& microwave frequencies. Indian journal of biochemistry \& biophysics, 21(1), 76.

Kumar, Y. R., \& Raju, G. (2014). Study of characteristics for dielectric properties of various biological tissues. International Journal of Advanced Research in Computer and Communication Engineering, Vol. 3, Issue 1.

Lazebnik, M., Popovic, D., McCartney, L., Watkins, C. B., Lindstrom, M. J., Harter, J. \& Temple, W. (2007). A large-scale study of the ultrawideband microwave dielectric properties of normal, benign and malignant breast tissues obtained from cancer surgeries. Physics in Medicine and Biology, 52(20), 6093.

Ortega-Palacios, R., Leija, L., Vera, A., \& Cepeda, M. F. J. (2010, September). Measurement of breast-tumor phantom dielectric properties for microwave breast cancer treatment evaluation. In Electrical Engineering Computing Science and Automatic Control (CCE), 2010 7th International Conference on (pp. 216-219). IEEE.

Tofighi, M. R., \& Daryoush, A. (2006). Measurement techniques for the electromagnetic characterization of biological materials. Engineering Electromagnetic Applications.

Venkatesh, M. S., \& Raghavan, G. S. V. (2005). An overview of dielectric properties measuring techniques. Canadian Biosystems Engineering, 47(7), 15-30. 